\title{
Behavioral mechanisms of context fear generalization in mice
}

\author{
Kylie A. Huckleberry, Laura B. Ferguson, and Michael R. Drew \\ Center for Learning and Memory, Department of Neuroscience, University of Texas at Austin, Austin, Texas 78712, USA
}

\begin{abstract}
There is growing interest in generalization of learned contextual fear, driven in part by the hypothesis that mood and anxiety disorders stem from impaired hippocampal mechanisms of fear generalization and discrimination. However, there has been relatively little investigation of the behavioral and procedural mechanisms that might control generalization of contextual fear. We assessed the relative contribution of different contextual features to context fear generalization and characterized how two common conditioning protocols-foreground (uncued) and background (cued) contextual fear conditioning-affected context fear generalization. In one experiment, mice were fear conditioned in context $A$, and then tested for contextual fear both in A and in an alternate context created by changing a subset of A's elements. The results suggest that floor configuration and odor are more salient features than chamber shape. A second experiment compared context fear generalization in background and foreground context conditioning. Although foreground conditioning produced more context fear than background conditioning, the two procedures produced equal amounts of generalized fear. Finally, results indicated that the order of context tests (original first versus alternate first) significantly modulates context fear generalization, perhaps because the original and alternate contexts are differentially sensitive to extinction. Overall, results demonstrate that context fear generalization is sensitive to procedural variations and likely reflects the operation of multiple interacting psychological and neural mechanisms.
\end{abstract}

[Supplemental material is available for this article.]

When a traumatic event occurs, the context typically becomes a conditioned stimulus (CS), and returning to the same or a similar context evokes fear. Overgeneralizing or inappropriately retrieving learned contextual fear may contribute to the pathogenesis of anxiety disorders. For instance, panic disorder is hypothesized to originate when the internal or external context in which a panic attack occurred becomes a CS, and the conditioned fear transfers-or generalizes-to other similar contexts (Lissek et al. 2010). Patients suffering from panic disorder display elevated fear generalization in a laboratory-based fear conditioning task, suggesting that excessive generalization may indeed underlie the disorder (Lissek et al. 2010). Such examples implicating fear generalization in pathogenesis of anxiety disorders have generated interest in understanding the neural and psychological mechanisms of fear generalization, particularly with respect to contexts (Sahay et al. 2011b; Kheirbek et al. 2012a).

Whereas fear conditioning to discrete cues such as simple tones can be supported by the amygdala and other subcortical structures, fear conditioning to a context typically recruits plasticity in both the amygdala and hippocampus. The hippocampus is hypothesized to form a conjunctive representation that binds together multiple elements of the context (Sutherland and Rudy 1989; Phillips and LeDoux 1992; Fanselow 2000). Post-training lesions to the hippocampus or its outputs impair expression of contextual fear, suggesting that hippocampal conjunctive encoding is the default learning strategy for contextual fear conditioning (CFC; Anagnostaras et al. 1999; Biedenkapp and Rudy 2009). CFC procedures evoke synchronized rhythmic activity between the amygdala and the hippocampus, suggesting coordinated mutual involvement in this form of learning (Seidenbecher et al. 2003). However, pretraining lesions to the hippocampus usually

\section{Corresponding author: drew@mail.clm.utexas.edu}

Article is online at http://www.learnmem.org/cgi/doi/10.1101/Im.042374.116. have little to no effect on CFC (Wiltgen et al. 2006; Biedenkapp and Rudy 2009), indicating that context fear learning can also be accomplished by hippocampus-independent mechanisms. In the absence of a functional hippocampus, animals may adopt an elemental strategy in which the context is encoded as a collection of independent elements or features (Anagnostaras et al. 2001).

Generalization occurs when a learned response is evoked by stimuli similar to the original CS. Although some level of generalization is adaptive because it allows a learned response to transfer to other relevant situations and stimuli, overgeneralization of fear can be debilitating. The neural mechanisms underlying generalization of fear are thus an area of intense investigation. Studies have implicated the hippocampus (Rudy and O'Reilly 1999) and more specifically, the hippocampal processes of pattern separation and pattern completion (Sahay et al. 2011b; Kheirbek et al. 2012a; Besnard and Sahay 2016) in controlling contextual fear generalization. For instance, the large number of cells and sparsity of firing within the dentate gyrus (DG) are believed to support pattern separation, which is hypothesized to limit context generalization by providing different contexts with more unique neural codes (Jung and McNaughton 1993; Leutgeb et al. 2007; McHugh et al. 2007; Nakashiba et al. 2012; Niibori et al. 2012). Consistent with this view, perturbations of DG function are associated with impaired context discrimination and increased context fear generalization (McHugh et al. 2007; Sahay et al. 2011a; Kheirbek et al. 2012b; Tronel et al. 2012; Wu and Hen 2014). Conversely, the strong auto-associative network of CA3 is thought

(C) 2016 Huckleberry et al. This article is distributed exclusively by Cold Spring Harbor Laboratory Press for the first 12 months after the full-issue publication date (see http://learnmem.cshlp.org/site/misc/terms.xhtml). After 12 months, it is available under a Creative Commons License (AttributionNonCommercial 4.0 International), as described at http://creativecommons. org/licenses/by-nc/4.0/. 
to support pattern completion, the process by which partial cues can evoke retrieval of a complete memory. Impairing CA3 function can also alter fear generalization (Cravens et al. 2006).

Despite the extensive recent work on the neural mechanisms of generalization, there has been relatively little investigation of behavioral and sensory influences on context generalization. For instance, CFC can be produced through background (cued) procedures, in which context conditioning occurs as a consequence of pairings between a discrete conditioned stimulus (CS) and shock, and foreground (uncued) procedures in which the context is the only explicit CS. Although these procedures are hypothesized to recruit different learning mechanisms (Phillips and LeDoux 1994; Calandreau et al. 2005; Trifilieff et al. 2006), their effects on context generalization have not been explicitly compared. Furthermore, while it is generally believed that rodents remember multiple features of conditioning contexts, to our knowledge, the relative contribution of particular context features to generalization and discrimination has not been investigated. Here we characterize context fear generalization in male C57BL/6J mice. After establishing two discriminable contexts, we compare the relative contribution of specific contextual features to context fear generalization. We also compare the extent to which background and foreground context fear conditioning engender generalized contextual fear. Finally, we explore the effect of test order (alternate first versus original first) on generalized context freezing.

\section{Results}

\section{Experiment 1: Establishing discriminable contexts}

First, we sought to establish two highly discriminable contexts and to confirm that freezing behavior in each context was attributable to the context-shock training rather than to intrinsic (nonbackground contextual conditioning in context $\mathrm{A}(n=10)$ or $\mathrm{B}$ $(n=10)$, and then received test sessions in both A and B in counterbalanced order (Fig. 1A). Mice froze significantly more in their training context than in the alternate context, regardless of the identity of each ( $P$ 's $\leq 0.004$; Fig. 1B). Data were analyzed using a three-way ANOVA with Training Context (A versus B) and Test Order (Original and Alternate versus Alternate and Original) as between-subject factors and Test Context (Original versus Alternate) as a within-subject factor. The analysis confirmed a significant effect of Test Context $\left(F_{(1,16)}=32.20, P<0.001\right)$, but the other main effects and interactions did not reach significance (full results are reported in Supplemental Table 1). This result confirms that Contexts A and B are discriminable and that freezing in each context is associative in nature. For comparison with Experassociative) features of the context. Mice received three-shock

iments 2 and 3, Supplemental Figure 1 shows context test performance as a function of test order (Original-Alternate versus Alternate-Original), which is discussed further below.

\section{Experiment 2: Which contextual features support context fear generalization?}

Context B was created by altering Context A in three ways: inserting a triangular roof, changing the floor configuration, and changing the scent. Experiment 2 was designed to assess the extent to which each of these changes contributed to context fear generalization. Mice received three-shock background contextual fear conditioning in Context A followed by two tests of contextelicited fear. One test occurred in A and the other in an altered context, in counterbalanced order. There were four alternate contexts, each created by changing a single feature of A (Roof $[n=7]$, Floor $[n=8]$, or Scent $[n=8]$ ) or by changing all three features (RFS; $n=12$; Fig. 2A).

The data for the context tests were analyzed using a threeway ANOVA with Alternate Context (RFS, F, S, or R), and Context Order $(\mathrm{A} \rightarrow$ Alternate versus Alternate $\rightarrow \mathrm{A})$ as betweensubjects factors and Test Context (A versus Alternate) as a within-subject factor. The full ANOVA results are reported in Supplemental Table 2. The ANOVA detected a significant Alternate Context $\times$ Test Context interaction $\left(F_{(3,27)}=4.46, P=\right.$ $0.011)$, confirming that the different alternate contexts elicited different levels of generalized fear. Our a priori predictions were that animals would freeze more in the original than the alternate context. Thus, within each group, planned comparisons were used to compare freezing in $\mathrm{A}$ and in the altered context (Fig. 2B). In groups Floor and RFS, freezing in A exceeded freezing in the altered context $\left(P^{\prime} \mathrm{S} \leq 0.003\right)$; in groups Scent $(P=0.137)$ and Roof $(P=$ 0.482 ), the freezing levels did not differ. The data indicate that changing only the floor was sufficient to render the two contexts discriminable, whereas changing only the scent or the roof may not be sufficient to support discriminated freezing.

The overall ANOVA also detected a significant Context Order $\times$ Test Context interaction $\left(F_{(1,27)}=10.18, P=0.004\right)$, indicating that the order of context tests influenced generalization between $\mathrm{A}$ and the alternate contexts. Post hoc comparisons indicated that freezing to A exceeded alternate context freezing when A was tested first $(P<0.001)$ but not when A was tested second $(P=0.556)$. Other pairwise comparisons did not reach significance. Experiment 3 will address potential explanations for this order effect.

To more explicitly compare the effectiveness of the different contextual alterations in promoting generalized freezing, we computed a discrimination ratio by expressing context $\mathrm{A}$ freezing as a proportion of summed freezing to both $\mathrm{A}$ and the alternate context $[\mathrm{A} /(\mathrm{A}+\mathrm{Alt})$.$] .$
A

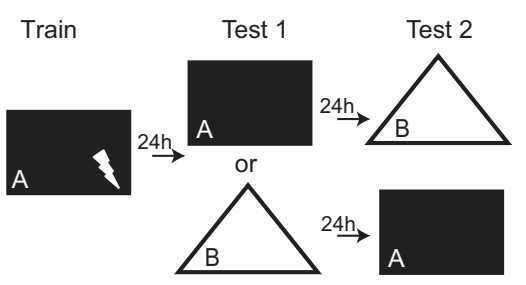

B

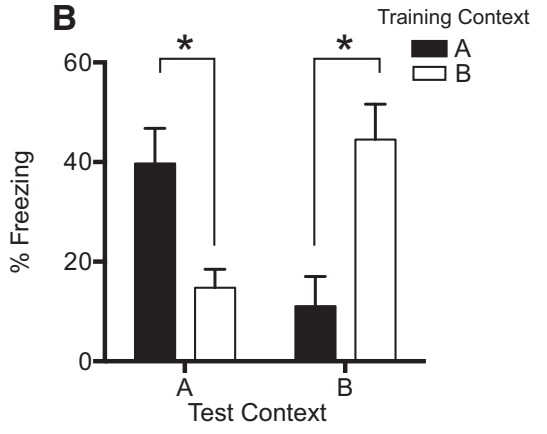

Figure 1. Establishing discriminable contexts. $(A)$ Mice were trained in either Context $\mathrm{A}(n=10)$ or Context $\mathrm{B}(n=10)$ and tested in both contexts in counterbalanced order. (B) Mean freezing to the test contexts. Error bars represent \pm 1 SEM. $\left(^{*}\right) P<0.05$. A higher discrimination ratio indicates less generalized freezing to the alternate context (Fig. 2C). One-way ANOVA comparing the discrimination ratios among the four alternate contexts was significant $\left(F_{(3,31)}=4.487, P=0.010\right)$. Pairwise comparisons indicated that RFS exceeded Roof $(P=0.006)$ and that the difference between Scent and Roof approached significance $(P=0.083)$. All other comparisons failed to reach significance $\left(P^{\prime} \mathrm{s} \geq\right.$ 0.215 ). We also evaluated our a priori prediction that the discrimination ratios should exceed the 0.5 chance level. $T$-tests revealed that the discrimination ratios for Groups RFS $(P<0.001)$, Floor 
A
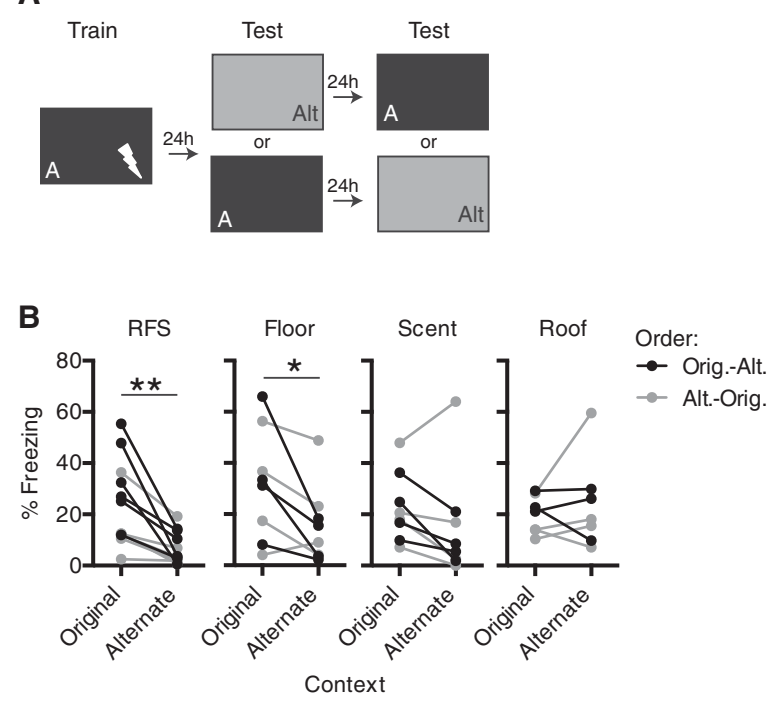

C

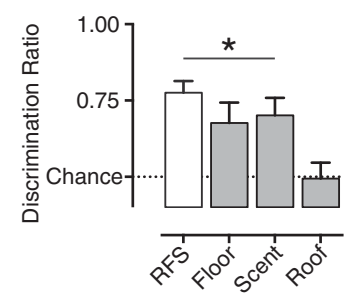

Figure 2. Determining the extent to which different contextual features contribute to context fear generalization. $(A)$ Mice were trained in Context A and tested both in the original context and an alternate context. Alternate contexts were created by changing either a single feature of Context A (Roof $[n=7]$, Floor $[n=8]$, or Scent $[n=8]$ ), or all three features (RFS; $n=12$ ). (B) Individual freezing data during test sessions as a function of alternate context. Mice froze more in Context A than in Floor or RFS $\left(P^{\prime} s \leq 0.003\right)$, but freezing did not differ between Context $A$ and Scent or Roof $\left(P^{\prime} s \geq 0.137\right)$. Black dots/lines indicate Context $A$ was tested first, and gray dots/lines indicate the Alternate Context was tested first. (C) Discrimination ratios $[\mathrm{A} /(\mathrm{A}+\mathrm{Alt})$.$] as a function of alternate context.$ Discrimination was better than chance $(0.5)$ for the RFS $(P<0.001)$, Floor $(P=0.033)$, and Scent $(P=0.018)$ alternate contexts but did not exceed chance for the Roof alternate context $(P=0.919)$. Gray line at 0.5 denotes chance performance. Error bars represent \pm 1 SEM. $\left({ }^{*}\right) P<0.05$.

$(P=0.033)$, and Scent $(P=0.018)$ exceeded 0.5 , whereas the ratio for Group Roof $(P=0.919)$ did not.

In summary, changing either the floor configuration or the scent was sufficient to produce discriminated freezing, whereas changing the roof only was not sufficient to support discriminated freezing. Although the effect of the scent change did not reach significance in all of our analyses, the effect is validated in Experiment 3, which had more power. The results suggest that the visual characteristics of a context, which were changed drastically by insertion of the roof, may exert a smaller influence on context fear generalization than do the tactile (floor) and olfactory characteristics. However, we are unable to rule out the possibility that the visual components of the floor changes also contributed to discrimination.

\section{Experiment 3: Fear generalization after foreground versus background context conditioning}

Next, we asked whether the two major categories of context conditioning procedures are differentially conducive to fear generali- zation. Context fear can be produced by background context conditioning, in which the US is explicitly paired with a discrete auditory or visual CS, and by foreground context conditioning, in which the context is the only explicit CS. These procedures typically generate different levels of contextual fear (Phillips and LeDoux 1994) and have been hypothesized to recruit different neural mechanisms (Phillips and LeDoux 1994; Calandreau et al. 2005; Trifilieff et al. 2006). In this experiment, we compared context fear generalization after either foreground or background context conditioning. Because foreground conditioning generates more contextual fear than background conditioning, we included two foreground conditioning procedures. One procedure (three-shock foreground conditioning) was equated with background conditioning with respect to the number and timing of shocks. The other procedure (one-shock foreground conditioning) was equated with background conditioning in terms of the amount of contextual fear produced.

An additional aim of Experiment 3 was to systematically explore the effects of test order on context freezing. In the previous experiments, we observed a consistent trend toward higher alternate context freezing when the alternate context was tested before rather than after the original context (Fig. 2B; Supplemental Table 2). However, the previous experiments did not provide sufficient statistical power to investigate this effect. Experiment 3 permitted us to analyze how test order affects freezing in both the original and alternate contexts.

Mice were trained using the three-shock background (Background; $n=23$ ), three-shock foreground (Foreground $\times 3$; $n=24$ ), or one-shock foreground (Foreground $\times 1 ; n=24$ ) contextual conditioning procedure in Context A (Fig. 3A), and context-elicited fear was tested in both $\mathrm{A}$ and an alternate context, in counterbalanced order (Fig. 3A,B). The alternate context was either Context RFS or Context Scent (S), as described in Experiment 2. On the basis of Experiment 2, Context $\mathrm{S}$ was expected to elicit significantly more generalized freezing than Context RFS.

Our analysis focused on mean freezing during the test sessions in the original and alternate contexts (Supplemental Fig. 2). These data were analyzed using ANOVA with Training Protocol, Alternate Context (RFS versus S), and Context Order $(\mathrm{A} \rightarrow$ Alternate versus Alternate $\rightarrow \mathrm{A}$ ) as between-subjects factors and Test Context (A versus Alternate) as a within-subject factor. The full ANOVA results are reported in Supplemental Table 3. The ANOVA detected significant effects of Training Protocol, the Test Context $\times$ Alternate Context interaction, and the Test Context $\times$ Context Order interaction. These effects are discussed in turn in the following paragraphs.

The significant main effect of Training Protocol indicates that Foreground $\times 3$ conditioning produced higher levels of freezing than the other protocols (Fig. 3C,D). Pairwise post hoc comparisons confirmed that mice receiving Foreground $\times 3$ training froze more in both the original and alternate contexts than did mice receiving either of the other two protocols $\left(P^{\prime} s \leq 0.001\right)$. Importantly, Training Protocol did not interact with any of the other variables ( $\left.P^{\prime} \mathrm{s}>0.278\right)$, including Test Context $(F<1)$, indicating that Training Protocol did not significantly affect the extent to which fear generalized between the original and alternate contexts. That is, the elevated freezing observed in the Foreground $\times 3$ group was no larger in the alternate contexts than in the original context. To further explore whether Training Protocol influenced context fear generalization, we computed discrimination ratios as in Experiment 2 (Supplemental Fig. 3). The effects of Training Protocol and Alternate Context on discrimination ratios were analyzed using ANOVA, which yielded a highly significant effect of Alternate Context $\left(F_{(1,65)}=22.650, P<\right.$ $0.0001)$, confirming that generalization between Contexts A and $S$ was stronger than that between Contexts A and RFS. The effects 
A
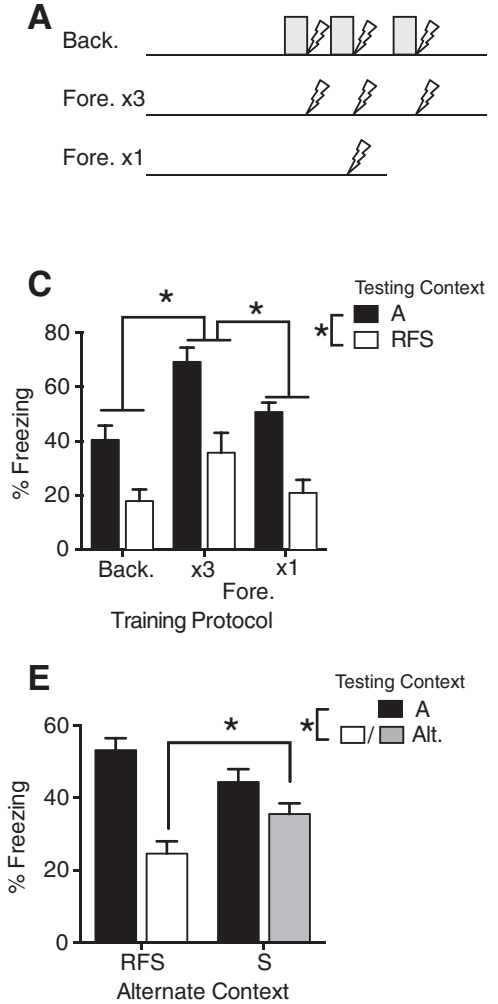

B
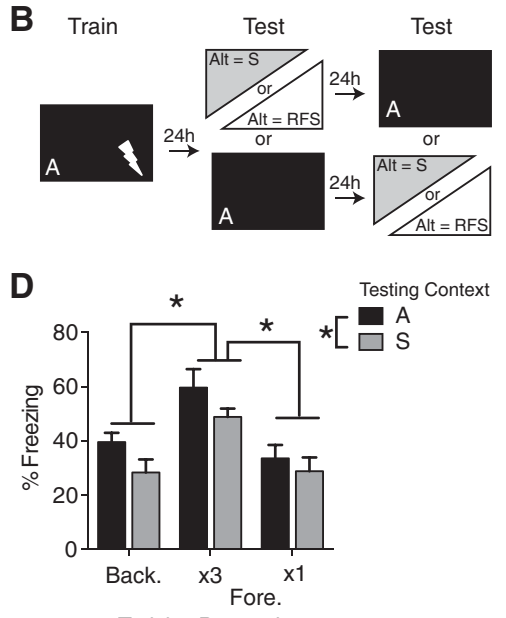

Training Protocol

$\mathbf{F}$

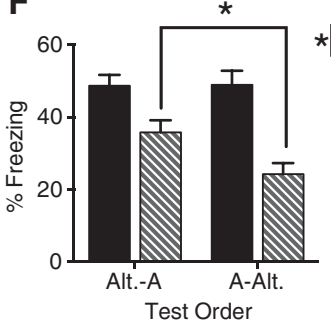

Testing Context

$\star[\mathrm{A}$

Figure 3. Fear generalization as a function of conditioning procedure. $(A)$ Three training procedures were compared in Experiment 3. Three-shock foreground conditioning (Fore. $\times 3 ; n=24$ ) was equated with background conditioning (Back.; $n=23$ ) with respect to the number and timing of shocks, while one-shock foreground conditioning (Fore. $\times 1 ; n=24$ ) was equated with background conditioning in terms of the amount of contextual fear produced. (B) Mice were trained in Context $A$ and tested in both $A$ and an alternate context (S or RFS). (C) Mean freezing during test sessions for mice tested with alternate context RFS as a function of training procedure. Mice froze more in A than in RFS, and Foreground $\times 3$ conditioning produced higher levels of freezing than did the other procedures $\left(P^{\prime} s \leq 0.001\right)$. (D) Mean freezing during test sessions for mice tested with alternate context $S$ as a function of training procedure. Mice froze more in A than in S, and Foreground $\times 3$ conditioning produced higher levels of freezing than did the other procedures $\left(P^{\prime} s \leq 0.001\right)$. (E) Mean freezing during test sessions as a function of alternate context. Pairwise post hoc comparisons confirmed that freezing was higher in Context $S$ than in Context RFS $(P=0.042)$, but freezing in Context A did not differ between groups $(P=0.125)$. $(F)$ Mean freezing during test sessions as a function of test order. Pairwise post hoc comparisons confirmed that freezing in the alternate contexts was lower in mice receiving the $A \rightarrow$ Alt. order than in those receiving the Alt $\rightarrow$ A order $(P=0.033)$, whereas freezing in $A$ was not affected by test order $(P=0.998)$. Error bars represent \pm 1 SEM. $\left({ }^{*}\right) P<0.05$.

of Training Protocol $\left(F_{(2,65)}<1\right)$ and the interaction $\left(F_{(2,65)}<1\right)$ were not significant, supporting the conclusion that Training Protocol did not affect context generalization.

The ANOVA of the freezing data also yielded a significant Test Context (A versus Alt.) $\times$ Alternate Context (RFS versus S) interaction (Fig. 3E). Pairwise post hoc comparisons confirmed that freezing was higher in the $S$ alternate context than in the RFS alternate context $(P=0.042)$, but freezing in the original context did not differ between groups that received different alternate contexts $(P=0.125)$.

Finally, the ANOVA on the freezing data yielded a significant Test Context (A. versus Alt) $\times$ Order $(\mathrm{A} \rightarrow$ Alt. versus Alt $\rightarrow \mathrm{A})$ interaction (Fig. $3 \mathrm{~F}$ ), suggesting that the order of context tests had a stronger effect on alternate context freezing than on original context freezing. Pairwise post hoc comparisons confirmed that freezing in the alternate contexts was lower in mice receiving the $\mathrm{A} \rightarrow$ Alt. order than in those receiving the Alt $\rightarrow$ A order $(P=0.033)$, whereas freezing in A was not affected by test order $(P=0.998)$. This finding may indicate that any fear extinction caused by the first context test has a stronger effect on subsequent alternate context freezing than on subsequent original context freezing.

In summary, Experiment 3 indicates that foreground conditioning produces more freezing than background conditioning (when the number of shocks is equal), but these two training procedures produce approximately equal amounts of generalized context freezing. In addition, results provide evidence of an asymmetrical effect of test order. Whereas freezing in the alternate context was higher when the alternate context was tested first than when it was tested second after the original context, freezing in the original context was less affected by test order.

\section{Discussion}

We characterized how sensory features of fear conditioning influence the generalization of conditioned fear from a trained context to an alternate context. We found that changing three physical features-shape, floor configuration, and scent-was sufficient to generate two distinct contexts that generated little crossgeneralization of fear. We compared the contributions of each of these three features to context fear generalization. Mice displayed complete generalization when two contexts differed only by the addition of a roof that altered the chamber shape, color, and lighting level. If two contexts differed only in floor configuration or scent, mice discriminated between the contexts, suggesting that floor configuration and scent are more salient contextual features than context shape. The relative effectiveness of the floor (tactile and visual) and ineffectiveness of the roof (primarily visual) as contextual cues are consistent with findings in the conditioned place preference paradigm indicating that tactile cues are more salient than visual cues as CSs for rodents (Cunningham et al. 2006).

Our experiments also assessed whether two common varieties of context conditioning-background and foregroundare differentially conducive to context fear generalization. Foreground and background conditioning have been hypothesized to recruit distinct neural mechanisms under some circumstances. For instance, pretraining lesions to the hippocampus have been reported to impair acquisition of background context fear conditioning while leaving foreground context fear conditioning intact (Phillips and LeDoux 1994; Gerlai 1998). The data have given rise to the idea that background conditioning favors formation of conjunctive context representations, which require the hippocampus (O'Reilly and Rudy 2001). Conversely, foreground conditioning may permit use of hippocampus-independent elemental representations, in which only a small number of experienced context features are remembered (Fanselow 1986). There is some indirect evidence that elemental context coding is more conducive to context fear generalization than is 
conjunctive encoding. Although pretraining lesions to the hippocampus do not prevent acquisition of context fear, context fear acquired in the absence of a hippocampus is overgeneralized (Frankland et al. 1998; Antoniadis and McDonald 2000). Similarly, as time passes after acquisition, context fear memories become hippocampus-independent (Anagnostaras et al. 1999) and context fear generalization increases (Biedenkapp and Rudy 2007; Wiltgen and Silva 2007; Wiltgen et al. 2010). If it is true that extrahippocampal context memories are coded elementally, then the data from these earlier papers suggest that elemental encoding may be more conducive to generalization than is conjunctive encoding.

The current experiments did not support this hypothesis that background and foreground conditioning promote different context encoding strategies. Although Foreground $(\times 3)$ conditioning produced more context fear than did background conditioning, the two procedures produced approximately equal amounts of context generalization. These data suggest that in mice with intact hippocampi at the time of fear acquisition, background and foreground context conditioning recruit similar learning mechanisms. Consistent with this idea, post-training hippocampal lesions impair both foreground (Wiltgen et al. 2006) and background (Anagnostaras et al. 1999) context conditioning, suggesting that rodents with intact hippocampi at the time of acquisition preferentially use the hippocampus-dependent conjunctive encoding strategy regardless of the conditioning procedure (Fanselow 2010). The primacy of conjunctive encoding is also supported by the finding that the immediate shock deficit is rescued by preexposure to the conditioning context but not by preexposure to individual elements of the context (Rudy and O'Reilly 1999). Presumably background and foreground conditioning produce different amounts of context conditioning (Fig. 3; Phillips and LeDoux 1994) not because they recruit different learning mechanisms but because the discrete CS in background conditioning competes with the context for associative strength (Rescorla and Wagner 1972) and/or attention (Mackintosh 1975), thereby reducing context conditioning in that procedure.

Our final observation was an effect of test order on context generalization, such that generalization was stronger when the alternate context was tested first than when it was tested after the original context, an effect observed in both Experiments 2 and 3 . Experiment 3 suggests that the order effect is primarily attributable to a change in freezing to the alternate context: namely, alternate context freezing was lower when it was tested second than when it was tested first. Notably, the order effect was asymmetrical: freezing to the alternate but not to the original context was affected by test order. The reduced freezing in the second context test may reflect extinction caused by the first context test in the original context. Generalized conditioned responses have been reported to extinguish more rapidly than conditioned responses to a trained CS (Hovland 1937). Alternatively, reexposure to the original context during the first test might reinforce the precision of the memory of the original context. In fact, Winocur et al. (2009) demonstrated that reexposure to the original but not to a distinct context $28 \mathrm{~d}$ after training enhanced rats' ability to discriminate by reactivating the hippocampus-dependent memory.

From a learning perspective, we can envision two accounts of the asymmetric order effect. An account based on an elemental model of context learning would assume that each alternate context elicits freezing by virtue of sharing some of A's elements, each of which has associative strength. During exposure to the alternate context, each of these shared elements undergoes extinction. Because the shared elements represent only a fraction of A's associative value, exposure to the alternate context has less effect on A's associative value than does exposure to A itself. Conversely, exposure to A would have a comparatively larger effect on alternate context freezing because exposure to A extinguishes all the shared elements from which the alternate context derives its associative value. Although this account appears compatible with our data, other lines of research (discussed above) favor the hypothesis that context representations are conjunctive rather than elemental.

A conjunctive account of extinction generalization was discussed by Bouton et al. (2012) in a paper reporting an asymmetrical extinction effect similar to what is reported here. Using an appetitive conditioning procedure with tone and light cues as CSs, Bouton et al. demonstrated that extinction transfers more readily from a compound stimulus to one of its elements than from an element to a compound. Although that effect could be explained using an elemental model, the authors also discussed a conjunctive account, based around the idea that unexpected exposure to an element independent from its compound evokes the formation of a new conjunctive representation. Thus, when an element is presented alone during extinction, this new configuration becomes associated with nonreinforcement, thereby protecting the original compound from extinction. Conversely, when the compound itself is extinguished, the extinction attaches to the original conjunctive representation. According to this hypothesis, testing in the original context would extinguish the original context representation, whereas testing in the similar context might evoke the formation of a new conjunctive representation associated with safety. The idea that extinction training can evoke the formation of new context representations is compatible with evidence that both tone and contextual fear extinction involve plasticity not only in brain regions thought to control associative value but also within hippocampal circuits believed to generate context memory representations (Corcoran and Maren 2001; Tronson et al. 2009; Wang et al. 2015). Regardless of the mechanism, our data suggest that test order can have a significant effect on context generalization, particularly when highly similar contexts are used. Moreover, when multiple context tests are administered to the same animal, generalized freezing may reflect a mixture of context fear generalization and context fear extinction.

Recent studies have begun to identify the neural mechanisms underlying fear generalization. Recordings in the lateral amygdala (LA) following discrimination training to two discrete, simple cues identified separate populations of neurons that signaled either generalized or cue-specific associations. Increasing generalized fear by increasing the US intensity altered the proportions of these cell populations, and manipulations increasing the excitability of LA neurons increased generalized fear (Ghosh and Chattarji 2015). Meanwhile, in CFC, generalization is regulated by both hippocampal and prefrontal mechanisms. Pattern separation in the DG is thought to be important for generating unique ensemble representations of similar contexts (Leutgeb et al. 2007). Indeed, context discrimination is impaired by perturbations to the DG, including knockout of DG NMDAR function (McHugh et al. 2007), ablation of adult hippocampal neurogenesis (Sahay et al. 2011a; Nakashiba et al. 2012; Niibori et al. 2012; Tronel et al. 2012), and knockout of NMDARs in adult-born neurons (Kheirbek et al. 2012b). Recently, a circuit comprising prefrontal cortex, thalamic nucleus reuniens (NR), and hippocampal CA1 was implicated in bidirectionally modulating context fear generalization. Silencing of prefrontal inputs to the NR or the NR inputs to hippocampal CA1 increased context fear memory generalization, whereas tonic activation of the NR neurons during fear acquisition reduced context fear generalization (Xu and Südhof 2013). The data suggest that fear generalization is regulated by multiple mechanisms within the amydala, thalamus, prefrontal cortex, and hippocampus. However, previous studies have not explicitly analyzed potential effects of test order, nor have they 
parametrically varied differences between the original and alternate contexts. Thus, there remains the question whether the identified neural mechanisms are dedicated to regulating fear specificity or, instead, modulate factors that contribute indirectly to observed fear generalization. For instance, in the $\mathrm{Xu}$ and Sudhof study, the alternate context was always tested after the original context, leaving open the possibility that the PFCNR-CA1 circuit modulates resistance to extinction rather than generalization per se.

The asymmetrical extinction effect we observed in Experiment 3 may be relevant to clinical treatments for disorders like PTSD that are characterized by inappropriate responses to contextual cues (Levy-Gigi et al. 2015). Exposure therapy is commonly used to treat PTSD and other anxiety disorders. For instance, virtual reality devices are being used to treat combat veterans by simulating contexts associated with trauma (Rothbaum et al. 1999; Reger et al. 2011). Although our experiments did not address extinction or exposure therapy per se, our data suggest that exposure to a context different from the trauma contexteven if the two are very similar-may be relatively ineffective in attenuating fear of the trauma context. This idea is in line with work suggesting that exposure therapy in a context different from-albeit similar to-a trauma context may not be effective unless it includes all of the salient stimuli (Andreano et al. 2009). However, our data also suggest that if all the salient conditioned stimuli are included in an extinction treatment, then generalized fear will be greatly reduced, thereby enhancing the efficacy of therapy.

Together our results identify procedural variables that influence the amount of contextual fear generalization and suggest that context generalization is a multidetermined process. The amount of generalization observed in any given experiment is likely to reflect a complex interaction among the contextual stimuli used, the order of context tests, and extinction produced by repeated testing. As a result, context generalization is likely to recruit multiple interacting neural mechanisms.

\section{Materials and Methods}

\section{Subjects}

One hundred and twenty-six male C57BL/6J mice aged 8-11 wk were used. Mice were group-housed (four per cage maximum) in plastic cages with wood chip bedding and maintained on a 12-h light-dark cycle. Food and water were available ad libitum. All procedures were approved by the University of Texas at Austin's Institutional Animal Care and Use Committee.

\section{Apparatus}

Training and testing took place during the light cycle in a room lighted with red bulbs. Fear conditioning was conducted in Med Associates conditioning chambers $(30.5 \mathrm{~cm}$ wide $\times 24.25 \mathrm{~cm}$ deep $\times 21.5 \mathrm{~cm}$ high) with two aluminum sidewalls, a Plexiglas door and ceiling, and a white vinyl back wall. These chambers were housed within a larger, sound-attenuating chamber equipped with a fan providing $\sim 65 \mathrm{~dB}$ ambient noise. During all procedures, chambers were illuminated from above with white light (141 lux) and infrared light. An infrared digital camera mounted on the side of the outer chamber recorded animal behavior during all sessions.

The conditioning chambers could be configured as two distinct contexts, A and B (aka RFS). Context A consisted of a straight stainless steel rod floor ( 36 stainless steel rods evenly spaced $8 \mathrm{~mm}$ from center to center) with a white paper towel covering the waste tray underneath. It was cleaned and scented with 70\% ethanol. Context B had a floor comprised of steel rods in staggered configuration (36 stainless steel rod floor spaced $8 \mathrm{~mm}$ from center to center with alternating heights), a brown paper towel in the waste tray, and a black, acrylic triangular roof placed over the floor. The addition of the roof created a tent shape, and the "tent" walls intersected the floor at an angle of $50^{\circ}$. Addition of the roof also reduced the peak height of the chamber to $18 \mathrm{~cm}$ and the ambient white light to 11 lux. Context B was cleaned and scented with Clorox Fresh Scent antiseptic wipes.

In addition to Contexts $\mathrm{A}$ and $\mathrm{B}$, three intermediate contexts were created by altering a single feature of Context A. In the "Floor" context only the floor was changed (Context A's floor and white paper towel were replaced with the staggered floor and brown paper towel). In the "Roof" context, the triangular roof was placed inside of Context A. In the "Scent" Context A's scent was changed (the chamber was cleaned and scented with Clorox Fresh Scent wipes rather than 70\% ethanol).

\section{Behavioral procedures}

\section{General}

All mice were gently handled for $\sim 2$ min per day for $5 \mathrm{~d}$ prior to experimentation. Mice were transported from the vivarium to a dimly lit holding room adjacent to the testing room a minimum $1 \mathrm{~h}$ prior to experimentation. For each session, mice were moved individually to and from the testing room in opaque white containers with clear lids. These containers were cleaned with 70\% ethanol between uses.

The testing procedures occurred over 3 consecutive days. Conditioning took place on day 1 . Mice received 3-min context test sessions in the original (A) and alternate contexts on days 2 and 3. The order of these test sessions was counterbalanced, such that half of all subjects received the original context test first and half received the alternate context test first. Because toneelicited fear was not of interest in these experiments, the tone was not presented during any of the test sessions.

\section{Contextual fear conditioning protocols}

Training for three-shock background (cued) CFC consisted of three tone-shock pairings during a $300 \mathrm{sec}$ session. The tone (5000 Hz, $85 \mathrm{~dB}$ tone) was presented for $20 \mathrm{sec}$ at 121, 160, and $220 \mathrm{sec}$ into the session. Each tone coterminated with a foot shock (1 sec, $0.75 \mathrm{~mA}$ ). The training protocol for three-shock foreground (uncued) CFC was identical to the three-shock background CFC protocol, except tones were not presented. In one-shock foreground CFC, training consisted of a single shock $(2 \mathrm{sec}, 0.75$ $\mathrm{mA}$ ) occurring $180 \mathrm{sec}$ into the 212-sec session (Wiltgen et al. 2001; Drew et al. 2010; Bernier et al. 2014).

\section{Statistical analysis}

Freezing was quantified with a pixel-change algorithm based on videos recorded at 30 frames/sec (VideoFreeze, Med Associates Inc.). Statistical analysis was performed using Prism 6 (GraphPad software) and JMP PRO 11 (SAS Institute Inc.). Significant interaction effects were probed using pairwise Sidak's multiple comparison tests (post hoc comparisons) or $t$-tests (planned comparisons). Alpha was set at 0.05 for all analyses.

\section{Acknowledgments}

Funding was provided by National Institutes of Health Grants R01 MH102595, R21 EY026446, and R00 MH083943 and UT Brain grant UTS-NNRI 365289.

\section{References}

Anagnostaras SG, Maren S, Fanselow MS. 1999. Temporally graded retrograde amnesia of contextual fear after hippocampal damage in rats: within-subjects examination. J Neurosci 19: 1106-1114.

Anagnostaras SG, Gale GD, Fanselow MS. 2001. Hippocampus and contextual fear conditioning: recent controversies and advances. Hippocampus 11: $8-17$. 
Andreano J, Liang K, Kong L, Hubbard D, Wiederhold BK, Wiederhold MD. 2009. Auditory cues increase the hippocampal response to unimodal virtual reality. Cyberpsychol Behav 12: 309-313.

Antoniadis EA, McDonald RJ. 2000. Amygdala, hippocampus and discriminative fear conditioning to context. Behav Brain Res 108: 1-19.

Bernier BE, Lacagnina AF, Drew MR. 2014. Potent attenuation of context fear by extinction training contiguous with acquisition. Learn Mem 22: $31-38$.

Besnard A, Sahay A. 2016. Adult hippocampal neurogenesis, fear generalization, and stress. Neuropsychopharmacology Rev 41: 24-44.

Biedenkapp JC, Rudy JW. 2007. Context preexposure prevents forgetting of a contextual fear memory: implication for regional changes in brain activation patterns associated with recent and remote memory tests. Learn Mem 14: 200-203.

Biedenkapp JC, Rudy JW. 2009. Hippocampal and extrahippocampal systems compete for control of contextual fear: role of ventral subiculum and amygdala. Learn Mem 16: 38-45.

Bouton ME, Doyle-Burr C, Vurbic D. 2012. Asymmetrical generalization of conditioning and extinction from compound to element and element to compound. J Exp Psychol Anim Behav Process 38: 381-393.

Calandreau L, Desmedt A, Decorte L, Jaffard R. 2005. A different recruitment of the lateral and basolateral amygdala promotes contextual or elemental conditioned association in pavlovian fear conditioning. Learn Mem 12: 383-388.

Corcoran KA, Maren S. 2001. Hippocampal inactivation disrupts contextual retrieval of fear memory after extinction. J Neurosci 21: $1720-1726$.

Cravens CJ, Vargas-Pinto N, Christian KM, Nakazawa K. 2006. CA3 NMDA receptors are crucial for rapid and automatic representation of context memory. Eur J Neurosci 24: 1771-1780.

Cunningham CL, Patel P, Milner L. 2006. Spatial location is critical for conditioning place preference with visual but not tactile stimuli. Behav Neurosci 120: 1115-1132.

Drew MR, Denny CA, Hen R. 2010. Arrest of adult hippocampal neurogenesis in mice impairs single- but not multiple-trial contextual fear conditioning. Behav Neurosci 124: 446-454.

Fanselow MS. 1986. Associative vs topographical accounts of the immediate shock-freezing deficit in rats: implications for the response selection rules governing species-specific defensive reactions. Learn Motiv 17: 16-39.

Fanselow MS. 2000. Contextual fear, gestalt memories, and the hippocampus. Behav Brain Res 110: 73-81.

Fanselow MS. 2010. From contextual fear to a dynamic view of memory systems. Trends Cogn Sci 14: 7-15.

Frankland PW, Cestari V, Filipkowski RK, McDonald RJ, Silva AJ. 1998. The dorsal hippocampus is essential for context discrimination but not for contextual conditioning. Behav Neurosci 112: 863-874.

Gerlai R. 1998. Contextual learning and cue association in fear conditioning in mice: a strain comparison and a lesion study. Behav Brain Res 95: 191-203.

Ghosh S, Chattarji S. 2015. Neuronal encoding of the switch from specific to generalized fear. Nat Neurosci 18: 112-120.

Hovland CI. 1937. The generalization of conditioned responses: III. Extinction, spontaneous recovery, and disinhibition of conditioned and generalized responses. J Exp Psychol 21: 47-62.

Jung MW, McNaughton BL. 1993. Spatial selectivity of unit activity in the hippocampal granular layer. Hippocampus 3: 165-182.

Kheirbek MA, Klemenhagen KC, Sahay A, Hen R. 2012a. Neurogenesis and generalization: a new approach to stratify and treat anxiety disorders. Nat Neurosci 15: 1613-1320.

Kheirbek MA, Tannenholz L, Hen R. 2012b. NR2B-dependent plasticity of adult-born granule cells is necessary for context discrimination. $J$ Neurosci 32: 8696-8702.

Leutgeb JK, Leutgeb S, Moser M-B, Moser EI. 2007. Pattern separation in the dentate gyrus and CA3 of the hippocampus. Science 315: 961-966.

Levy-Gigi E, Szabo C, Richter-Levin G, Kéri S. 2015. Reduced hippocampal volume is associated with overgeneralization of negative context in individuals with PTSD. Neuropsychology 29: 151-161.

Lissek S, Rabin S, Heller RE, Lukenbaugh D, Geraci M, Pine DS, Grillon C. 2010. Overgeneralization of conditioned fear as a pathogenic marker of panic disorder. Am J Psychiatry 167: 47-55.

Mackintosh NJ. 1975. A theory of attention: variations in the associability of stimuli with reinforcement. Psychol Rev 82: 276-298.

McHugh TJ, Jones MW, Quinn JJ, Balthasar N, Coppari R, Elmquist JK, Lowell BB, Fanselow MS, Wilson MA, Tonegawa S. 2007. Dentate gyrus NMDA receptors mediate rapid pattern separation in the hippocampal network. Science 317: 94-99.

Nakashiba T, Cushman JD, Pelkey KA, Renaudineau S, Ruhl DL, McHugh TJ, Barrera VR, Chittajallu R, Iwamoto KS, McBain CJ, et al.
2012. Young dentate granule cells mediate pattern separation, whereas old granule cells facilitate pattern completion. Cell 149: 188-201.

Niibori Y, Yu T-S, Epp JR, Akers KG, Josselyn SA, Frankland PW. 2012. Suppression of adult neurogenesis impairs population coding of similar contexts in hippocampal CA3 region. Nat Commun 3: 1253.

O'Reilly RC, Rudy JW. 2001. Conjunctive representations in learning and memory: principles of cortical and hippocampal function. Psychol Rev 108: $311-345$

Phillips RG, LeDoux JE. 1992. Differential contribution of amygdala and hippocampus to cued and contextual fear conditioning. Behav Neurosci 106: $274-285$

Phillips RG, LeDoux JE. 1994. Lesions of the dorsal hippocampal formation interfere with background but not foreground contextual fear conditioning. Learn Mem 1: 34-44.

Reger GM, Holloway KM, Candy C, Rothbaum BO, Difede J, Rizzo AA, Gahm GA. 2011. Effectiveness of virtual reality exposure therapy for active duty soldiers in a military mental health clinic. J Trauma Stres 24: $93-96$.

Rescorla RA, Wagner AR. 1972. A theory of Pavlovian conditioning: Variations in the effectiveness of reinforcement and nonreinforcement. In Classical Conditioning II (ed. Black AH, Prokasy WF), pp. 64-99. Appleton-Century-Crofts.

Rothbaum BO, Hodges L, Alarcon R, Ready D, Shahar F, Graap K, Pair J, Hebert P, Gotz D, Wills B, et al. 1999. Virtual reality exposure therapy for PTSD Vietnam Veterans: a case study. J Trauma Stress 12: 263-271.

Rudy JW, O'Reilly RC. 1999. Contextual fear conditioning, conjunctive representations, pattern completion, and the hippocampus. Behav Neurosci 113: 867-880.

Sahay A, Scobie KN, Hill AS, O'Carroll CM, Kheirbek MA, Burghardt NS, Fenton AA, Dranovsky A, Hen R. 2011a. Increasing adult hippocampal neurogenesis is sufficient to improve pattern separation. Nature 472: 466-470.

Sahay A, Wilson DA, Hen R. 2011b. Pattern separation: a common function for new neurons in hippocampus and olfactory bulb. Neuron 70: $582-588$.

Seidenbecher T, Laxmi TR, Stork O, Pape H-C. 2003. Amygdalar and hippocampal theta rhythm synchronization during fear memory retrieval. Science 301: 846-850.

Sutherland RJ, Rudy JW. 1989. Configural association theory: the role of the hippocampal formation in learning, memory, and amnesia. Psychobiology 17: 129-144.

Trifilieff P, Herry C, Vanhoutte P, Caboche J, Desmedt A, Riedel G, Mons N, Micheau J. 2006. Foreground contextual fear consolidation requires two independent phases of hippocampal ERK/CREB activation. Learn Mem 13: 349-358.

Tronel S, Belnoue L, Grosjean N, Revest J-M, Piazza P-V, Koehl M, Abrous DN. 2012. Adult-born neurons are necessary for extended contextual discrimination. Hippocampus 22: 292-298.

Tronson NC, Schrick C, Guzman YF, Huh KH, Srivastava DP, Penzes P, Guedea AL, Gao C, Radulovic J. 2009. Segregated populations of hippocampal principal CA1 neurons mediating conditioning and extinction of contextual fear. J Neuorsci 29: 3387-3394.

Wang ME, Yuan RK, Keinath AT, Ramos Alvarez MM, Muzzio IA. 2015. Extinction of learned fear induces hippocampal place cell remapping. J Neurosci 35: 9122-9136.

Wiltgen BJ, Silva AJ. 2007. Memory for context becomes less specific with time. Learn Mem 14: 313-317.

Wiltgen BJ, Sanders MJ, Behne NS, Fanselow MS. 2001. Sex differences, context preexposure, and the immediate shock deficit in pavlovian context conditioning with mice. Behav Neurosci 115: 26-32.

Wiltgen BJ, Sanders MJ, Anagnostaras SG, Sage JR, Fanselow MS. 2006. Context fear learning in the absence of the hippocampus. J Neurosci 26: $5484-5491$.

Wiltgen BJ, Zhou M, Cai Y, Balaji J, Karlsson MG, Parivash SN, Li W, Silva AJ. 2010. The hippocampus plays a selective role in the retrieval of detailed contextual memories. Curr Biol 20: 1336-1344.

Winocur G, Frankland PW, Sekeres M, Fogel S, Moscovitch M. 2009. Changes in context-specificity during memory reconsolidation: selective effects of hippocampal lesions. Learn Mem 16: 722-729.

Wu MV, Hen R. 2014. Functional dissociation of adult-born neurons along the dorsoventral axis of the dentate gyrus. Hippocampus 24: 751-761.

Xu W, Südhof TC. 2013. A neural circuit for memory specificity and generalization. Science 339: 1290-1295.

Received March 21, 2016; accepted in revised form September 20, 2016. 


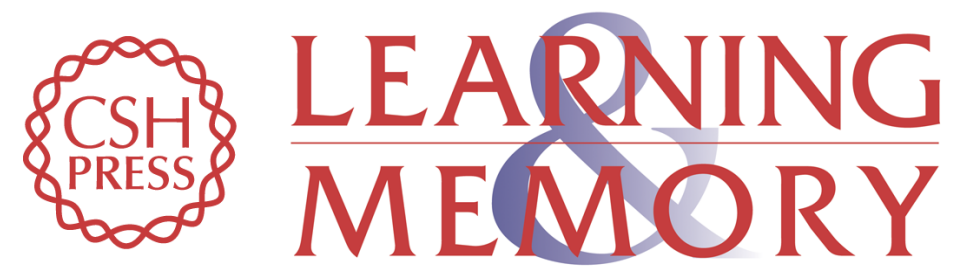

\section{Behavioral mechanisms of context fear generalization in mice}

Kylie A. Huckleberry, Laura B. Ferguson and Michael R. Drew

Learn. Mem. 2016, 23:

Access the most recent version at doi:10.1101//m.042374.116

Supplemental http://learnmem.cshlp.org/content/suppl/2016/11/09/23.12.703.DC1
Material

References This article cites 52 articles, 17 of which can be accessed free at: http://learnmem.cshlp.org/content/23/12/703.full.html\#ref-list-1

Creative This article is distributed exclusively by Cold Spring Harbor Laboratory Press for the Commons first 12 months after the full-issue publication date (see

License http://learnmem.cshlp.org/site/misc/terms.xhtml). After 12 months, it is available under a Creative Commons License (Attribution-NonCommercial 4.0 International), as described at http://creativecommons.org/licenses/by-nc/4.0/.

Email Alerting Receive free email alerts when new articles cite this article - sign up in the box at the Service top right corner of the article or click here. 